\title{
MERALGIA PARESTHETICA CAUSED BY THORACOLUMBAR BRACE IN CONSERVATIVELY TREATED THORACOLUMBAR FRACTURES
}

\author{
(1) İsmail Bozkurt \\ Çankırı State Hospital, Clinic of Neurosurgery, Çankırı, Turkey
}

Objective: Lateral femoral cutaneous neuropathy or meralgia paresthetica (MP) occurs for various reasons. MP developing after spine surgery is not uncommon and is mostly due to the prone position during surgery or iliac graft harvesting. However, it is usually overlooked due to mild symptoms and a self-limiting course. The purpose of this study was to present a case series of five patients who were followed up with conservative treatment for vertebral fractures and developed MP after prolonged use of lumbar braces.

Materials and Methods: The sample comprised five patients with thoracolumbar fractures who did not meet the surgical criteria of the Thoracolumbar Injury Classification and Severity score and who were advised to use lumbar braces for 8 weeks and to return to the outpatient department. These patients did not present for follow-up due to the coronavirus disease-2019 (COVID-19) pandemic and continued to use the lumbar brace for more than the advised 8 weeks. A retrospective evaluation of age, sex, body mass index (BMI), comorbidities, and duration of brace use was conducted.

Results: Three of the five patients were male with an average age of $61 \pm 18$ years, average BMI of $29.3 \pm 4.8 \mathrm{~kg} / \mathrm{m}^{2}$, (after excluding the young and normal-weight patient, the average age and BMI increased to $70 \pm 5$ years and $31.6 \pm 1.5 \mathrm{~kg} / \mathrm{m}^{2}$, respectively), and an average brace use duration of $18.4 \pm 3.2$ weeks. Three patients presented with MP on the left side.

Conclusion: Although tight-fitting pants/corsets/belts/body armor can cause MP, no cases of MP caused by lumbar braces used for conservative treatment of vertebral fractures have been reported. This case series arose from the effects of COVID-19, as the patients wanted to stay home.

Keywords: Meralgia paresthetica, brace, COVID-19, fracture, vertebra

\section{INTRODUCTION}

Meralgia paresthetica (MP) was first defined by Bernhardt and Roth in 1895 as a neurological disorder causing pain or dysesthesia in the anterolateral aspect of the thigh caused by compression of the lateral femoral cutaneous nerve (LFCN). A Dutch population study reported an incidence rate of MP of 0.43 per 10,000 person-years, whereas an older study reported 3 per 10,000 general clinic patients, with a predominance between the ages of 30 and 50 years ${ }^{(1)}$.

The LFCN courses through the pelvis adjacent to the lateral side of the iliopsoas muscle and enters the thigh through or under the inguinal ligament close to the anterior superior iliac spine where entrapment occurs most frequently. As the nerve is purely sensory, symptoms relating to sensorial dysfunction, such as burning, tingling, or pins, are the most frequent clinical findings ${ }^{(2)}$. MP predominantly arises from mechanical entrapment of the LFCN, and causes include trauma, tight- fitting pants/corsets/belts/body armor, compression from carrying heavy objects supported on the thigh, and use of a wallet. latrogenic causes have been reported due to iliac bone grafting, prolonged lithotomy and the prone spine positions, injury while performing inguinal herniorrhaphy, and retractor compression during gastroplasty ${ }^{(2)}$. Obesity, pregnancy, and diabetes mellitus are risk factors ${ }^{(1)}$. However, two thin pediatric patients with MP have also been reported ${ }^{(3)}$.

The diagnosis is mostly clinical due to an unpleasant feeling at the site innervated by the LFCN. Physicians may employ electrophysiological testing but the reliability of somatosensoryevoked potentials (SSEPs) in MP is controversial ${ }^{(4)}$.The Thoracolumbar Injury Classification and Severity score (TLICS) was designed to take into account the morphology of the fracture, posterior ligamentous complex integrity, and neurological status to allow for better categorization and communication among surgeons ${ }^{(5)}$. The AO Spine Injury Classification system establishes subgroups from the original Magerl AO concept but uses three main injury categories ${ }^{(6)}$. Although various 
approaches exist to determine whether a conservative or surgical treatment course is appropriate, the overall agreement is to follow-up patients with a thoracolumbosacral orthosis (TLSO) brace for 8-12 weeks in neurologically intact cases that do not meet the instability criteria(7).

\section{MATERIALS AND METHODS}

The study was performed in agreement with the ethical standards specified in the Declaration of Helsinki and was accepted by the Research Ethics Committee of Çankırı Karatekin University (No: 464/010321). Approval from the Ministry of Health in regards to studies involving Coronavirus disease-2019 (COVID-19) cases was obtained (2021-01-11T14_56_04). A written informed consent from each patient approving for images and clinical information regarding to their case be reported in a medical publication was obtained. Patients with thoracolumbar fractures due to trauma who applied for followup after the COVID-19 pandemic began were analyzed. Patients who did not meet the surgical criteria, who were advised to use a TLSO for 8 weeks, and who were followed up with neurological and radiological evaluations were included. All corsets were provided by the same supplier, who was experienced and the only supplier in the city. The patients were checked for proper usage after the TLSO was applied. They were advised to use diclofenac potassium $50 \mathrm{mg} /$ day for persistent pain. All patients that delayed their follow-up examination and continued to use the TLSO and presented with a tingling, burning, or a numbness sensation in the anterolateral aspect of the thigh were included.

\section{Outcome Measures}

Demographic information was obtained along with the TLICS score, $\mathrm{AO}$ spine injury classification, $\mathrm{BMI}$, comorbidities, duration of TLSO usage, and the side of MP.

\section{RESULTS}

Five patients presented with dysesthesia in the anterolateral aspect of the thigh after the COVID-19 pandemic began and who used the TLSO for $18.4 \pm 3.2$ weeks instead of the advised
8 weeks. The patients had an average age of $61 \pm 18$ years and an average $\mathrm{BMI}$ of $29.3 \pm 4.8 \mathrm{~kg} / \mathrm{m}^{2}$. However, when the young and otherwise healthy patient no. 2 was excluded, the average age and $\mathrm{BMI}$ increased to $70 \pm 5$ years and $31.6 \pm 1.5 \mathrm{~kg} / \mathrm{m}^{2}$, respectively. Three patients had hypertension, and two patients had been diagnosed with type 2 diabetes previously. Three patients had a type A1 AO classification of vertebral fracture along with a TLICS score of 1 , and the other two had type A3 and a score of 1 . None of the patients were indicated for surgery at the time of the diagnosis or after their follow-up examination. All patients presented to the emergency ward after trauma with isolated vertebral fractures but only patients no. 1 and no. 4 presented with scalp abrasions and sutured lacerations due to high-energy traffic accidents.

All patients complained of aggravated symptoms at noon, particularly while standing, and symptoms were relieved after removing the brace before going to bed. The patients were relieved of their MP symptoms for an average of 5 days after discontinuing the TLSO. Only patient no. 1 had continuing symptoms and was prescribed $75 \mathrm{mg}$ pregabalin twice daily for 28 days, which was discontinued after the symptoms disappeared. The patients' characteristics are summarized in Table 1. The computed tomography findings of each patient are shown in Figures 1-5.

\section{DISCUSSION}

A case series of five patients with thoracolumbar fractures who were treated conservatively with bracing are reported. These patients used the braces longer than the advised 8 weeks due to the COVID-19 pandemic. They were diagnosed with MP based on sensory deficit symptoms in the distribution area of the LFCN without motor deficits. Although the use of tight-fitting pants, corsets, belts, and body armor can cause MP, a literature review did not yield any reports of MP associated with thoracolumbar bracing.

$M P$ is a purely sensory disorder involving the LFCN derived from the L2-L3 nerve root. The most common compression site is where the nerve passes through or under the inguinal

Table 1. List of patients and attributes

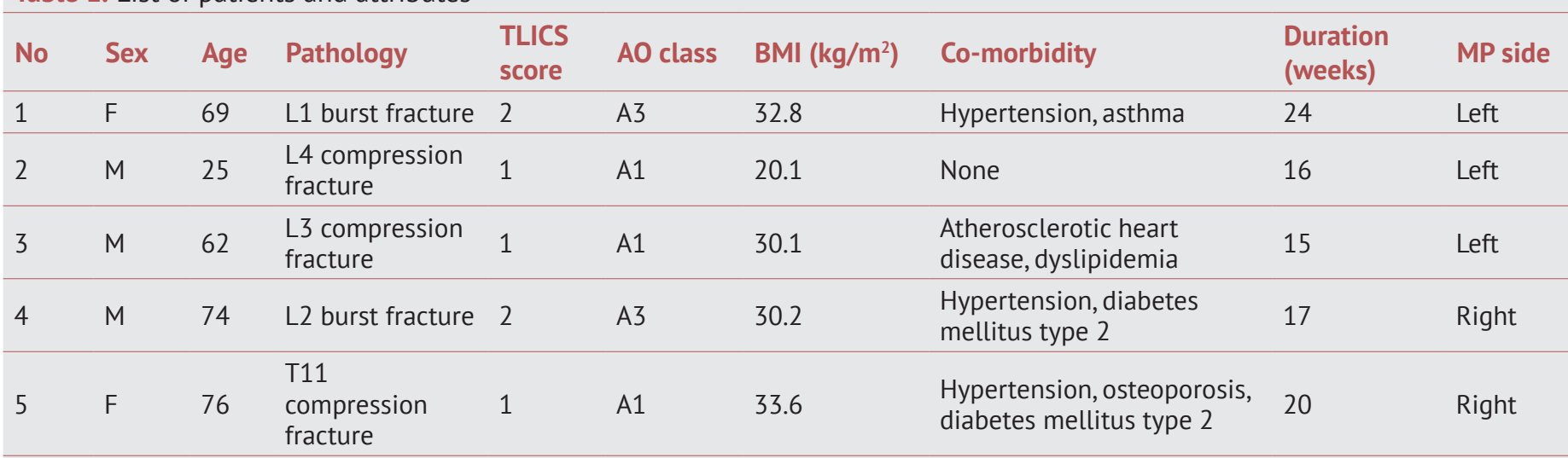

TLICS: Thoracolumbar Injury Classification and Severity score, BMI: Body mass index, MP: Meralgia paresthetica 


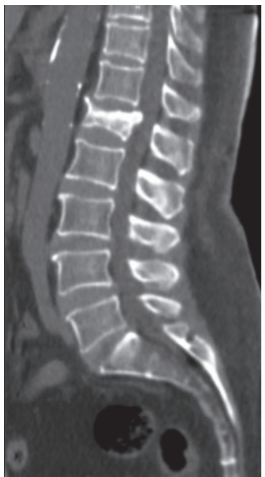

1

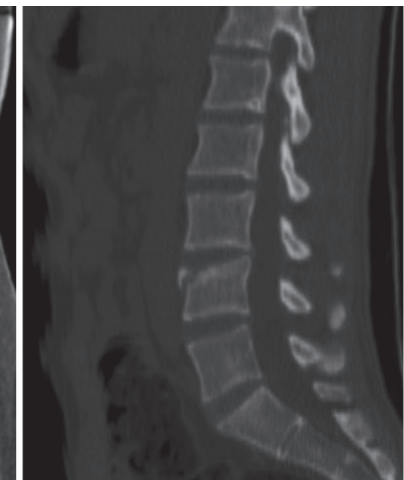

2

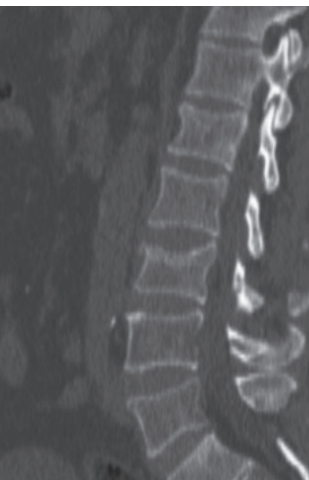

3

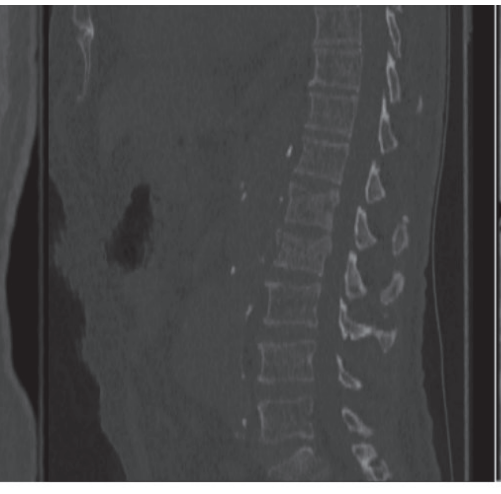

4

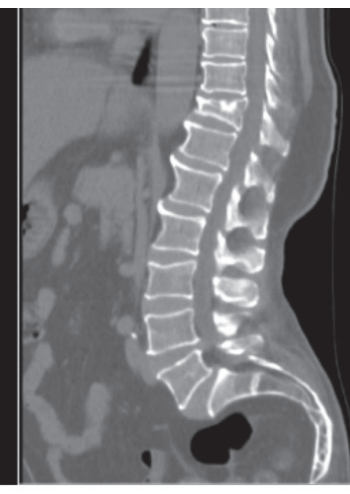

5

Figure 1-5. The sagittal reconstructed view of thoracolumbar CT findings as discussed in Table 1

$\mathrm{CT}$ : Computed tomography

ligament. Obesity or tight-fitting clothes and belts are risk factors for mechanical compression. The patients in this case series fit the diagnosis of MP, but no nerve conduction study was ordered due to low reliability ${ }^{(8)}$. Four of the five patients in this case series had an average BMI of $31.6 \mathrm{~kg} / \mathrm{m}^{2}$ (type 1 obesity). Two of the four patients were also diagnosed with type 2 diabetes. Both obesity and type 2 diabetes are risk factors for $M P^{(1)}$. However, one of the patients had a BMI of $20.1 \mathrm{~kg} / \mathrm{m}^{2}$, which is close to the lower level of normal weight. Although a low $\mathrm{BMI}$ is not a known risk factor, two thin pediatric patients with MP have been reported (3). Relatively thin people lack extra subcutaneous adipose tissue, allowing superficial nerves to course without extra protection, which would make them vulnerable to trauma and chronic compression.

The one factor that all patients had in common was the use of a TLSO. Although bracing is a low-risk and cost-effective method for treating thoracolumbar fractures, it does have limitations. Pressure sores due to rigid braces, non-compliance, diminished pulmonary capacity, and weakening of the loadbearing musculature are known complications $s^{(9)}$. The brace itself is a compressing factor around the abdomen and at the superior edge of the pelvis. While standing, the inferior border of the brace corresponds roughly to the superior onethird of the inguinal ligament, where the LFCN courses. While sitting with the brace, the inferior border completely rests on the waistline and the inguinal region. This may have caused compression of the LFCN and the symptoms. The patients in this case series were examined after the use of a TLSO and were properly trained to remove the appliance while lying down. However, no emphasis was placed on the significance of protecting the iliac wing and axilla from pressure, which should have been mentioned along with tips to protect against sores. The patients felt increased dysesthesia while standing upright, which exacerbates this syndrome ${ }^{(10)}$. The patients were unable to recall the exact timing of the symptoms, as most of them did not complain in the beginning. The suggested time frame for a TLSO is $8-12$ weeks. The patients in this case series used the brace for an average of 18.4 weeks. Four of the five patients in this case series were obese, which would make a difference. Patient no. 2 was thin, which allowed the brace's compressive force to directly press on the LFCN. In addition to these factors, the COVID-19 pandemic restricted social life and increased stay-at-home policies; thus, more sedentary time was spent at home with increased sitting time. This may be another contributing factor.

The patients in this case series were not evaluated via electromyography, as confounding results have been reported with this procedure. Although a study evaluating SSEPs between 20 patients and 22 healthy controls concluded that SSEPs are a diagnostic aid, another study demonstrated that SSEPs produce abnormal readings in the case of very serious nerve damage ${ }^{(1)}$. Performing electromyography is difficult in obese patients; thus, it should not be routinely employed or required for diagnosing MP but rather used to eliminate other differential diagnoses ${ }^{(2)}$.

\section{CONCLUSION}

Bracing is the proper approach to follow up a thoracolumbar fracture when surgery is not indicated. Previous data have revealed similar outcomes compared with surgical intervention. This study of five patients describes a complication of lumbar bracing that has not been reported previously. Perhaps a cotton coating on the inferior edge of the brace would help diminish this effect.

Although this is a case series of five patients who developed MP after prolonged use of a TLSO due to a thoracolumbar fracture, it provides evidence of an additional cause of MP that has not been reported. This report serves as a reminder to all physicians that the impact of the COVID-19 pandemic is much more prominent than anticipated, affecting the habits of all patients. The follow-up can be facilitated by phone calls. This case series of five patients had the limitations of being a retrospective analysis that was prone to selection bias and lacked a control arm. 


\section{Ethics}

Ethics Committee Approval: The study was performed in agreement with the ethical standards specified in the Declaration of Helsinki and was accepted by the Research Ethics Committee of Çankırı Karatekin University (number: 464/010321).

Informed Consent: A written informed consent from each patient approving for images and clinical information regarding to their case be reported in a medical publication was obtained.

Peer-review: Internally peer-reviewed.

Financial Disclosure: The author declared that this study received no financial support.

\section{REFERENCES}

1. Harney D, Patijn J. Meralgia paresthetica: diagnosis and management strategies. Pain Med. 2007;8:669-77.

2. David WS, Meralgia Paresthetica. In: Michael JA., Robert BD. (Eds.). Encyclopedia of the Neurological Sciences (Second Edition). Massachusetts General Hospital, United States of America 2014;pp:1099-100.
3. Richer LP, Shevell MI, Stewart J, Poulin C. Pediatric meralgia paresthetica. Pediatr Neurol. 2002;26:321-3.

4. Seror P. Lateral femoral cutaneous nerve conduction $v$ somatosensory evoked potentials for electrodiagnosis of meralgia paresthetica. Am J Phys Med Rehabil. 1999;78:313-6.

5. Lee JY, Vaccaro AR, Lim MR, Oner FC, Hulbert RJ, Hedlund R, et al. Thoracolumbar injury classification and severity score: a new paradigm for the treatment of thoracolumbar spine trauma. J Orthop Sci. 2005;10:671-5.

6. Reinhold M, Audigé L, Schnake KJ, Bellabarba C, Dai LY, Oner FC. AO spine injury classification system: a revision proposal for the thoracic and lumbar spine. Eur Spine J. 2013;22:2184-201.

7. Kim BG, Dan JM, Shin DE. Treatment of thoracolumbar fracture. Asian Spine J. 2015;9:133-46.

8. Fargo MV, Konitzer LN. Meralgia paresthetica due to body armor wear in U.S. soldiers serving in Iraq: a case report and review of the literature. Mil Med. 2007;172:663-5.

9. Chang V, Holly LT. Bracing for thoracolumbar fractures. Neurosurg Focus. 2014;37:E3. doi: 10.3171/2014.4.FOCUS1477

10. Shapiro BE, Preston DC. Entrapment and compressive neuropathies. Med Clin North Am. 2003;87:663-96. 